\title{
Grains of paradise (Aframomum melegueta) extract activates brown adipose tissue and increases whole-body energy expenditure in men
}

\author{
Jun Sugita ${ }^{1,2}$, Takeshi Yoneshiro ${ }^{3}$, Takuya Hatano ${ }^{1}$, Sayuri Aita ${ }^{1}$, Takeshi Ikemoto ${ }^{2}$, Hideyo Uchiwa $^{2}$, \\ Toshihiko Iwanaga $^{3}$, Toshimitsu Kameya ${ }^{4}$, Yuko Kawai ${ }^{4}$ and Masayuki Saito ${ }^{{ }^{*}}$ \\ ${ }^{1}$ Department of Nutrition, School of Nursing and Nutrition, Tenshi Collage, Kita-13, Higashi-3, Higashi-ku, \\ Sapporo 065-0013, Japan \\ ${ }^{2}$ Innovative Beauty Science Laboratory, Kanebo Cosmetics, Inc., Odawara 250-0002, Japan \\ ${ }^{3}$ Department of Anatomy, Hokkaido University Graduate School of Medicine, Sapporo 060-8638, Japan \\ ${ }^{4}$ LSI Sapporo Clinic, Sapporo 065-0013, Japan \\ (Submitted 13 July 2012 - Final revision received 29 October 2012 - Accepted 23 November 2012 - First published online 11 January 2013)
}

\section{Abstract}

Brown adipose tissue (BAT) is responsible for cold- and diet-induced thermogenesis, and thereby contributes to the control of whole-body energy expenditure (EE) and body fat content. BAT activity can be assessed by fluoro-2-deoxyglucose (FDG)-positron emission tomography (PET) in human subjects. Grains of paradise (GP, Aframomum melegueta), a species of the ginger family, contain pungent, aromatic ketones such as 6-paradol, 6-gingerol and 6-shogaol. An alcohol extract of GP seeds and 6-paradol are known to activate BAT thermogenesis in small rodents. The present study aimed to examine the effects of the GP extract on whole-body EE and to analyse its relation to BAT activity in men. A total of nineteen healthy male volunteers aged 20-32 years underwent FDG-PET after $2 \mathrm{~h}$ of exposure to cold at $19^{\circ} \mathrm{C}$ with light clothing. A total of twelve subjects showed marked FDG uptake into the adipose tissue of the supraclavicular and paraspinal regions (BAT positive). The remaining seven showed no detectable uptake (BAT negative). Within 4 weeks after the FDG-PET examination, whole-body EE was measured at $27^{\circ} \mathrm{C}$ before and after oral ingestion of GP extract $(40 \mathrm{mg})$ in a single-blind, randomised, placebocontrolled, crossover design. The resting EE of the BAT-positive group did not differ from that of the BAT-negative group. After GP extract ingestion, the EE of the BAT-positive group increased within $2 \mathrm{~h}$ to a significantly greater $(P<0 \cdot 01)$ level than that of the BAT-negative group. Placebo ingestion produced no significant change in EE. These results suggest that oral ingestion of GP extract increases wholebody EE through the activation of BAT in human subjects.

Key words: Grains of paradise: 6-Paradol: Brown adipose tissue: Energy expenditure

Brown adipose tissue (BAT) is the major site for sympathetically activated thermogenesis during cold exposure and spontaneous overfeeding, at least in small rodents ${ }^{(1)}$. Recent studies using fluoro-2-deoxyglucose (FDG)-positron emission tomography (PET) combined with computed tomography (CT) have revealed the existence of metabolically active BAT in adult human subjects ${ }^{(2-4)}$. It is now established that, in healthy adults, BAT is activated by acute cold exposure and significantly contributes to cold-induced thermogenesis ${ }^{(2-5)}$. It has also been demonstrated that the prevalence and activity of BAT are lower in subjects with higher adiposity, being negatively correlated with BMI, body fat content and visceral $\mathrm{fat}^{(2,3)}$. Moreover we found that the prevalence of BAT decreased with age, being more than $50 \%$ in the twenties, but less than $10 \%$ in the fifties and sixties, and that decreased
BAT activity is associated with age-related accumulation of body fat ${ }^{(6)}$. It is thus likely that BAT, based on its thermogenic activity, contributes to the control of whole-body energy expenditure (EE) and body fat metabolism in human subjects, as established in small rodents, and thereby is a promising target for interventions to prevent and treat obesity.

A number of food ingredients have been proposed as tools for increasing EE and decreasing body fat. One of these is capsaicin, the pungent ingredient of hot pepper, which activates the adreno-sympathetic nervous system and BAT, increases $\mathrm{EE}$ and fat oxidation and reduces body fat ${ }^{(7-11)}$. Recently, we reported that non-pungent capsaicin analogues (capsinoids) increase EE through the activation of BAT in human subjects $^{(12)}$, as they do in mice ${ }^{(13)}$. As the effects of capsaicin and capsinoids are much attenuated in mice deficient in

Abbreviations: BAT, brown adipose tissue; CT, computed tomography; EE, energy expenditure; FDG, fluoro-2-deoxyglucose; GP, grains of paradise; PET, positron emission tomography; TRPV1, transient receptor potential vanilloid 1 .

*Corresponding author: M. Saito, fax +81 11741 1077, email saito@tenshi.ac.jp 
transient receptor potential vanilloid 1 (TRPV1) ${ }^{(14)}$, a receptor for capsaicin, the thermic and fat-reducing effects of these compounds would be attributable to the activation of the pathway of TRPV1, the sympathetic nervous system and BAT.

The species known as 'grains of paradise' (Aframomum melegueta (Rosco) K. Schum.) (GP), also called Guinea pepper or Alligator pepper, is a member of the Zingiberaceae family native to West Africa. GP seeds are used as a spice for flavouring food and have a wide range of ethnobotanical uses, for example, as a remedy for treating stomach ache, diarrhoea and snake bite ${ }^{(15)}$. It has also been shown that aqueous seed extract has anti-inflammatory and peripheral analgesic activities $^{(16)}$. GP seeds are very rich in non-volatile pungent compounds such as 6-paradol, 6-gingerol, 6-shogaol and other related compounds ${ }^{(17-19)}$. Like capsaicin, these compounds contain a vanilloid moiety and activate TRPV1 ${ }^{(20)}$. Recently, Iwami et al. ${ }^{(21)}$ reported in rats that intragastric administration of an alcohol extract of GP and 6-paradol results in enhanced efferent discharges of sympathetic nerves to BAT and a significant rise in BAT temperature. Thus, it might be expected that GP extract, like capsaicin and capsinoids, activates BAT thermogenesis and increases $\mathrm{EE}$ in human subjects. To test this idea, in the present study, we examined the effects of GP-extract ingestion on EE by indirect calorimetry in healthy human volunteers and analysed its relation to BAT activity assessed by FDG-PET.

\section{Methods}

\section{Subjects}

A total of nineteen healthy male volunteers aged $20-32$ years were recruited and carefully instructed regarding the study, and gave their informed consent to participate in it. Each subject underwent a standardised health examination and FDG-PET/CT. The study was conducted according to the guidelines laid down in the Declaration of Helsinki and all the procedures were approved by the institutional review boards of Tenshi College. Written informed consent was obtained from all the subjects.

\section{Test substances}

Seeds of A. melegueta, commercially available as a spice produced in Côte d'Ivoire, were purchased from the Thiercelin Company. A single batch of dry GP seeds was soaked for extraction in five times volume of $95 \%$ ethanol for $24 \mathrm{~h}$ at reflux condition, with continuous agitation by a magnetic stirrer. The resulting ethanolic extract was filtrated and the solvent was removed under vacuum, yielding $4.2 \%$ of the GP seeds in weight. HPLC analysis of the extract revealed four major peaks, identified as 6-gingerol (15.2\%), 6-paradol (12.5\%), 6-shogaol (1.7\%) and 6-gingeredione (4.0\%). Other components included in the GP extract were caryophyllene and $\alpha$-humulene (5\%), TAG (20\%) and palmitic and oleic acids (3.2\%). A measure of $10 \mathrm{mg}$ of GP extract was mixed with $190 \mathrm{mg}$ of a mixture of rapeseed oil (158 mg) and beeswax (32 mg) in a capsule, which consisted of gelatin $(97.9 \mathrm{mg})$, glycerol $(34.3 \mathrm{mg})$ and caramel $(7.8 \mathrm{mg})$. The placebo capsule contained no GP extract. Preliminary experiments confirmed that daily oral ingestion of the test capsules (four capsules after breakfast, three capsules after lunch and three capsules after dinner) for 4 weeks caused no noticeable symptoms or adverse events.

\section{Fluoro-2-deoxyglucose-positron emission tomography/ computed tomography}

FDG-PET/CT examination was performed as described previously $^{(5)}$. Briefly, after fasting overnight, the subjects entered an air-conditioned room at $19^{\circ} \mathrm{C}$ with light clothing (usually a T-shirt with underwear), and put their feet on an ice block wrapped in cloth intermittently (usually for $4 \mathrm{~min}$ after every $5 \mathrm{~min}$ ). After $1 \mathrm{~h}$ in these cold conditions, they were given an intravenous injection of ${ }^{18} \mathrm{~F}-\mathrm{FDG}$ at doses of $1 \cdot 66-5 \cdot 18 \mathrm{MBq} / \mathrm{kg}$ body weight and continued under the same cold conditions. After $1 \mathrm{~h}$ of the ${ }^{18}$ F-FDG injection, PET/CT scans were performed employing a PET/CT system (Aquiduo; Toshiba Medical Systems) in a room at $24^{\circ} \mathrm{C}$.

PET and CT images were co-registered and analysed using a VOX-BASE workstation (J-MAC Systems). Two experienced, blinded observers assessed the FDG uptake, particularly on both sides of the neck and paravertebral regions, by visually judging the presence of radioactivity greater than that of the background. BAT activity in the neck region was quantified by calculating the maximal standardised uptake value, defined as the radioactivity per $\mathrm{ml}$ within the region of interest divided by the injected dose in MBq per g of body weight. For dividing subjects into BAT-positive and BAT-negative groups, a cutoff value of $2 \cdot 0$ was applied ${ }^{(6)}$.

\section{Anthropometric and body fat measurement}

BMI was calculated as the body weight in kg divided by the square of height in $\mathrm{m}\left(\mathrm{kg} / \mathrm{m}^{2}\right)$, and the percentage body fat was estimated by the multi-frequency bioelectric impedance method (InBody 320 Body Composition Analyzer; Biospace). The lean body mass was calculated as the difference between body weight and body fat mass.

\section{Indirect calorimetry}

Within 4 weeks after the FDG-PET/CT examination, the responses of whole-body $\mathrm{EE}$ to oral ingestion of either GP extract or placebo were tested in a single-blind, randomised crossover design with the BAT-positive and BAT-negative groups. The two tests were conducted 1-3 weeks apart.

Whole-body EE was estimated by the means of respiratory gas analyser connected to a ventilated hood (O-Jiro; Alko Systems). In brief, after fasting for 6-12 h, the subjects were asked to relax on a bed with light clothing in a room at $27^{\circ} \mathrm{C}$, and $\mathrm{O}_{2}$ consumption and $\mathrm{CO}_{2}$ production were continuously recorded for $30 \mathrm{~min}$. The stable value of the final 10-min period was used to calculate the resting EE and respiratory quotient. The subjects were then given four capsules of GP extract or placebo (a total dose of 40 or $0 \mathrm{mg}$, respectively) 
(a)

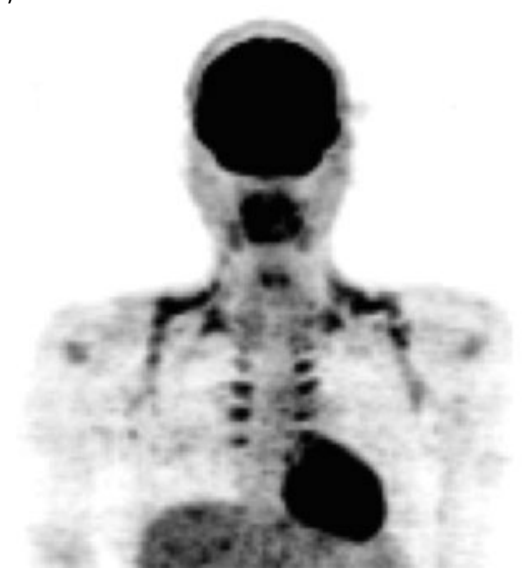

(b)

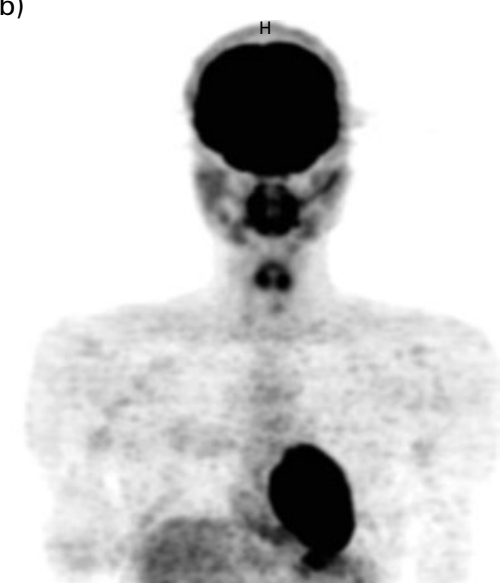

Fig. 1. Brown adipose tissue (BAT) detected by fluoro-2-deoxyglucose (FDG)-positron emission tomography (PET)/computed tomography (CT). FDG-PET/CT was conducted after $2 \mathrm{~h}$ cold exposure at $19^{\circ} \mathrm{C}$. In (a) (BAT positive), a marked uptake of FDG was noted in the adipose tissue in the supraclavicular and paraspinal regions, while in (b) (BAT-negative) no FDG uptake was detected in the same regions.

with $100 \mathrm{ml}$ water within the duration of 1 min. After 15, 45, 75 and $105 \mathrm{~min}$, respiratory gas parameters were recorded for $20 \mathrm{~min}$ each, and the $\mathrm{EE}$ and respiratory quotient were calculated for the final 10-min period.

\section{Data analysis}

Data are expressed as means with their standard errors and analysed by either $t$ test or three-factor ANOVA for repeated measures on two within-subject factors (time and GP/placebo) and one between-subject factor (BAT-positive/negative) with post hoc testing by Tukey's test using IBM SPSS Statistics 18.0 (IBM Japan). Values were considered to be statistically significant if $P<0.05$.

\section{Results}

Before the measurement of EE, all nineteen subjects had fasted overnight and were given an FDG-PET/CT examination after being in cold conditions at $19^{\circ} \mathrm{C}$ for $2 \mathrm{~h}$. Some subjects showed a clear and intense FDG uptake in adipose tissues in the supraclavicular and paraspinal regions, the maximal standardised uptake value being 3.2-31.4 (11.0 (SEM 2.6), whereas other subjects showed no detectable FDG uptake into the adipose tissues (Fig. 1). Based on these results, they were divided into BAT-positive $(n$ 12) and BAT-negative ( $n$ 7) groups. No significant difference between the two groups was found in terms of age, resting EE or anthropometric parameters such as BMI, body fat content and fat-free mass (Table 1).

Within 4 weeks after the FDG-PET/CT examination, subjects were asked to fast overnight and then underwent respiratory gas analysis (Fig. 2). In resting conditions before ingestion of the GP extract, the mean EE calculated from $\mathrm{O}_{2}$ consumption and $\mathrm{CO}_{2}$ production was 6163 (SEM 242) kJ/d for the BAT-positive group, not notably different from that for the BAT-negative group (6439 (SEM 209) kJ/d). In the BAT-positive group, EE increased at $0.5-1.5 \mathrm{~h}$ after ingestion of the GP extract
(40 mg), showing a maximal increase of 361 (SEM 86) kJ/d at $0.5 \mathrm{~h}$, while it changed little after placebo ingestion (Fig. 2). By contrast, in the BAT-negative group, no notable change was observed after ingestion of either the GP extract or placebo for at least $2 \mathrm{~h}$. The effects of GP extract and BAT on EE were analysed by three-factor ANOVA for repeated measures on two within-subject factors (time and GP/placebo) and one between-subject factor (BAT-positive/negative). Significant effects of time $(P<0.05), \mathrm{GP} \times \operatorname{BAT}(P<0.01)$ and time $\times \mathrm{GP} \times$ BAT $(P<0.01)$ were found. The significant interaction of time $\times$ GP $\times$ BAT implied that the difference in EE responses to GP and placebo was larger for the BAT-positive than for the BAT-negative group. Collectively, the EE response to GP extract in the BAT-positive group was significantly higher than that to the placebo group $(0.5$ and $1.5 \mathrm{~h})$ and also than to those in the BAT-negative group $(1.0$ and $1.5 \mathrm{~h})$. Fig. 3 shows the EE response to GP extract during the $2 \mathrm{~h}$ period expressed as AUC. It was $24.7(\operatorname{sem} 6 \cdot 1) \mathrm{kJ} / \mathrm{h}$ in the BAT-positive group, which was significantly greater than that in the BAT-negative group $(-3 \cdot 2(\operatorname{sem} 10 \cdot 1) \mathrm{kJ} / \mathrm{h}, P<0 \cdot 05)$ and also that following placebo ingestion $(3.0($ SEM 5.3$) \mathrm{kJ} / \mathrm{h}, P<0.01)$. Similar effects of GP extract ingestion were also confirmed when EE was calculated per kg fat-free mass (Figs. 2 and 3). Thus, the EE response to the GP extract was dependent on the presence of metabolically active BAT.

Table 1. Subjects' profiles

(Mean values with their standard errors)

\begin{tabular}{|c|c|c|c|c|}
\hline & \multicolumn{2}{|c|}{$\begin{array}{l}\text { BAT positive } \\
(n 12)\end{array}$} & \multicolumn{2}{|c|}{$\begin{array}{l}\text { BAT negative } \\
(n 7)\end{array}$} \\
\hline & Mean & SEM & Mean & SEM \\
\hline Age (years) & $23 \cdot 8$ & $1 \cdot 1$ & $21 \cdot 3$ & 0.3 \\
\hline $\mathrm{BMI}\left(\mathrm{kg} / \mathrm{m}^{2}\right)$ & 21.6 & 0.7 & $21 \cdot 0$ & 0.4 \\
\hline Body fat content (\%) & $15 \cdot 9$ & 1.5 & 14.7 & 1.0 \\
\hline Fat-free mass (kg) & $52 \cdot 4$ & 1.9 & $54 \cdot 1$ & $2 \cdot 0$ \\
\hline REE $(k J / d)$ & 6163 & 242 & 6493 & 209 \\
\hline
\end{tabular}

BAT, brown adipose tissue; REE, resting energy expenditure. 
(a)
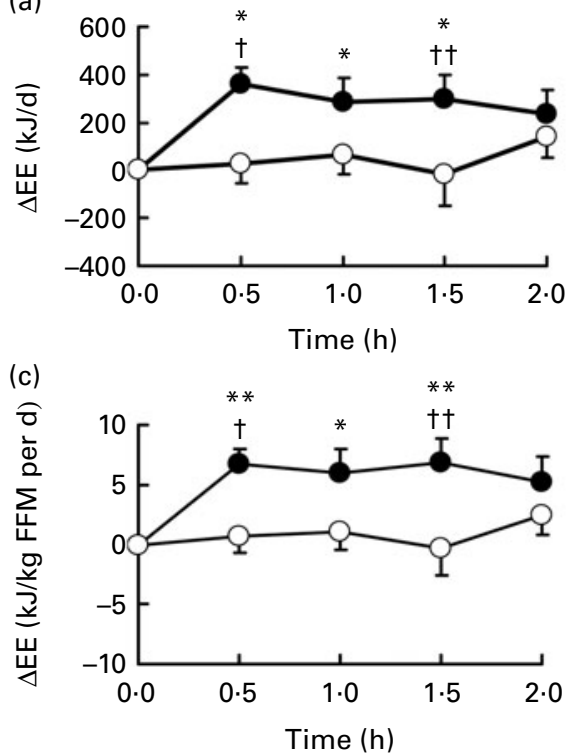

(b)

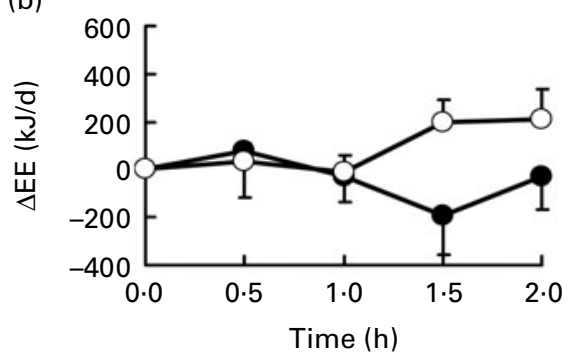

(d)

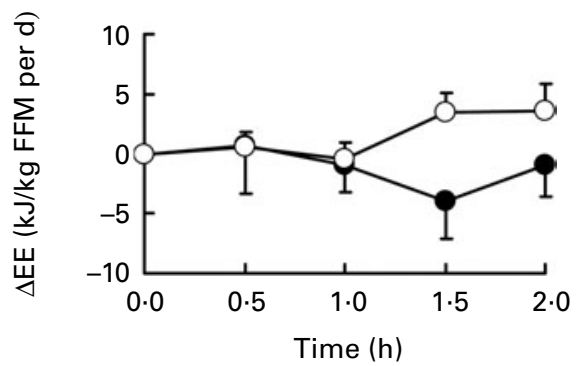

Fig. 2. Energy expenditure change $(\triangle \mathrm{EE})$ after oral ingestion of grains of paradise (GP) extract and placebo. $\Delta \mathrm{EE}$ before (0h) and after oral ingestion of $40 \mathrm{mg}$ GP extract (๑) or placebo (O). (a), (b) $\Delta \mathrm{EE}$, (c), (d) $\Delta \mathrm{EE}$ adjusted for fat-free mass (FFM), (a), (c) brown adipose tissue (BAT)-positive group ( $n$ 12), (b), (d) BAT-negative group $(n 7)$. Values are means with their standard errors represented by vertical bars. Mean values were significantly different compared with $0 \mathrm{~h}$ : ${ }^{*} P<0.05$ and ${ }^{* \star} P<0.01$. Mean values were significantly different compared with placebo: $† P<0.05$ and $\dagger+P<0.01$. ANOVA showed significant effects of time $(P<0.05)$, GP $\times$ BAT $(P<0.01)$ and time $\times$ GP $\times$ BAT $(P<0.01)$

The respiratory quotient was also calculated from $\mathrm{O}_{2}$ consumption and $\mathrm{CO}_{2}$ production (Fig. 4), and found not to be changed after GP extract and placebo ingestion at least during the $2 \mathrm{~h}$ period.

\section{Discussion}

As reported previously ${ }^{(2)}$, the metabolic activity of BAT can be assessed by FDG-PET/CT performed after acute cold exposure. In the present study, we applied this method for the detection of BAT in young male volunteers and divided them into two groups showing undetectable BAT (BAT negative) and metabolically active BAT (BAT positive). Consistent with our previous

(a)

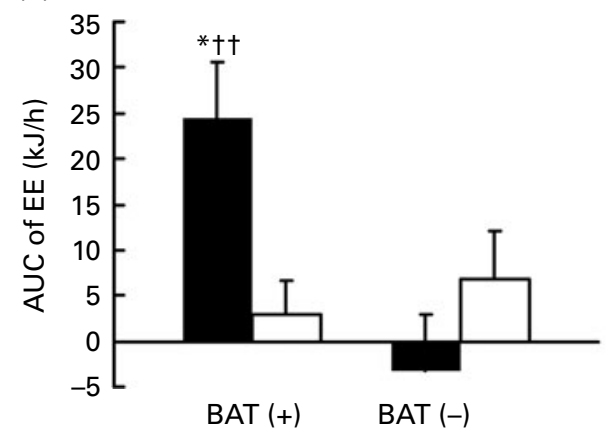

results $^{(5,6)}$, cold-activated BAT was detected in more than half the subjects. When compared with the BAT-negative group, the BAT-positive group showed comparable anthropometric parameters, including adiposity and resting EE. This allowed comparison of EE responses without consideration of the possible effects of adiposity on the responses.

The present results show that the oral ingestion of GP extract elicits a significant increase in EE in the BAT-positive group, while placebo ingestion shows no notable change. By contrast, neither GP extract, nor placebo ingestion changes $\mathrm{EE}$ in the BAT-negative group. Moreover, the effect of GP extract is stronger in the BAT-positive group than in the BAT-negative group. These results indicate that BAT is (b)

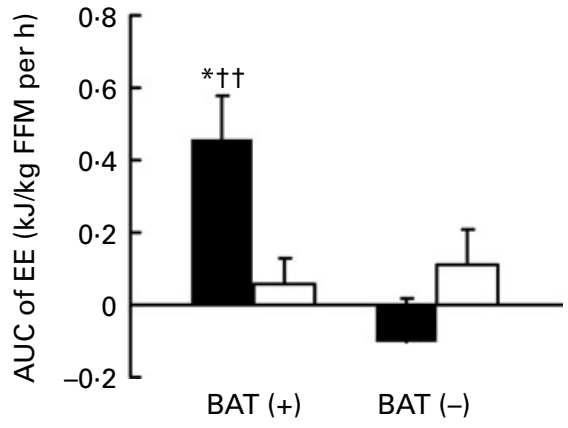

Fig. 3. Response of energy expenditure (EE) to grains of paradise (GP) extract. The response of EE to GP extract ( $\square$ ) and placebo ( $\square$ ) was calculated as the AUC between 0 and $2 \mathrm{~h}$ from the data in Fig. 1. (a) AUC as a whole, (b) AUC adjusted for fat-free-mass (FFM). Values are means with their standard errors represented by vertical bars. * Mean values were significantly different compared with the brown adipose tissue (BAT) negative $(P<0.05)$ group. $\dagger+$ Mean values were significantly different compared with the placebo $(P<0.01)$ group. 

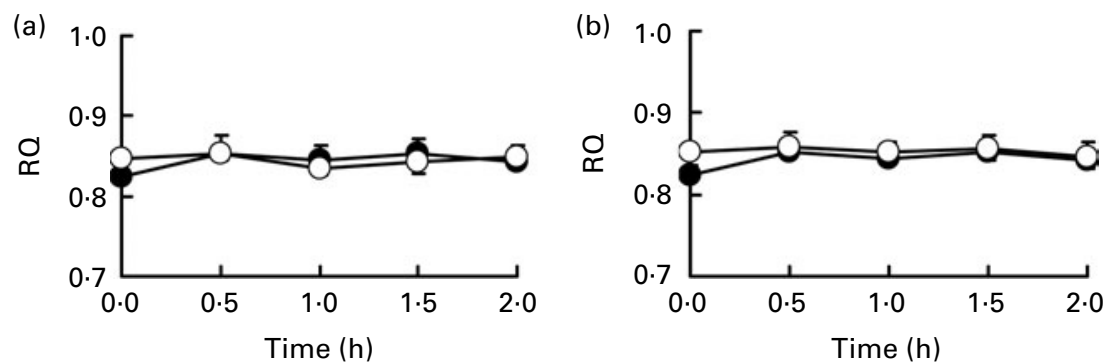

Fig. 4. Respiratory quotient $(R Q)$ after ingestion of grains of paradise (GP) extract and placebo. RQ after ingestion of GP extract $(\bullet)$ and placebo $(O)$ in (a) the brown adipose tissue (BAT)-positive and (b) the BAT-negative subjects is shown. Values are means with their standard errors represented by vertical bars.

involved in the GP-induced increase in EE. This seems consistent with a previous report on rats, in which GP extract activates BAT thermogenesis ${ }^{(21)}$.

As the test sample is an ethanol extract of GP seeds, the compounds responsible for the observed effect of GP extract are not known at present. The GP extract contained various compounds with a vanilloid moiety such as 6-paradol, 6-gingerol and 6-shogaol. All these compounds are capable of activating TRPV1, which is involved in the thermic effects of capsaicin and capsinoids. Potency in terms of activating TRPV1 is half-maximal effective concentration $\left(\mathrm{EC}_{50}\right)=0 \cdot 2 \mu \mathrm{M}$ for 6-shogaol and $\mathrm{EC}_{50}=0.7 \mu \mathrm{M}$ for 6-paradol, which are comparable with that of capsaicin and capsinoids and stronger than that of 6-gingerol $\left(\mathrm{EC}_{50}=3 \cdot 3 \mu \mathrm{M}\right)^{(20,22)}$. However, the content of 6 -shogaol $(1.7 \%)$ in the extract was much lower than that of 6-gingerol (15.2\%) and 6-paradol (12.5\%). Taken together, 6-paradol seems to be the most likely candidate responsible for the effects of GP extract observed in the present study. In fact, Iwami et al. ${ }^{(21)}$ reported that $5 \mathrm{mg}$ of 6-paradol activated sympathetic nerve activity in rats to a similar extent as $30 \mathrm{mg}$ of GP extract. In the present study, we used $40 \mathrm{mg}$ of GP extract which contained $5.0 \mathrm{mg}$ of 6-paradol. In addition to the vanilloid compounds, the GP extract also contained TAG and fatty acids, which may also activate BAT thermogenesis $^{(23)}$ and be responsible for the observed effects of GP extract. However, this is unlikely because the contents of TAG and fatty acids were almost similar in the GP and placebo capsules used in the present study.

The present results suggest that GP extract, like capsaicin and capsinoids, may be a potential tool for increasing BAT thermogenesis and decreasing body fat. In fact, based on preliminary experiments, we have observed that mice that were given either GP extract or 6-paradol showed lower body fat contents than those given placebo, without notable changes in food intake. Moreover, in human subjects, daily ingestion of GP extract for 4 weeks resulted in a slight but significant reduction in visceral fat. The present results support the hypothesis that the anti-obesity effect of GP extract depends on its stimulatory effect on BAT thermogenesis. In summary, GP extract can potentially increase EE through the activation of BAT in healthy men, and may, therefore, be useful for reducing body fat.

\section{Acknowledgements}

The author's contributions were as follows: T. Y. and M. S. designed the research, J. S., T. Y., T. H. and S. A. conducted the research, J. S., T. Y., T. I., H. U., T. I. and M. S. analysed the data, T. K. and Y. K. conducted FDG-PET/CT and M. S. wrote the manuscript and had primary responsibility for the final content. All authors have read and approved the final manuscript. The present study was supported in part by a Grant-in-Aid for Scientific Research from the Ministry of Education, Culture, Sports, Science and Technology of Japan (22590227). Kanebo Cosmetics, Inc. did not have any influence over the recruitment of subjects or on data collection, analysis and interpretation in the study. None of the authors has any conflicts of interest to declare.

\section{References}

1. Cannon B \& Nedergaard J (2004) Brown adipose tissue: function and physiological significance. Physiol Rev 84, $277-359$.

2. Saito M, Okamatsu-Ogura Y, Matsushita M, et al. (2009) High incidence of metabolically active brown adipose tissue in healthy adult humans: effects of cold exposure and adiposity. Diabetes 58, 1526-1531.

3. Van Marken Lichtenbelt WD, Vanhommerig JW, Smulders NM, et al. (2009) Cold-activated brown adipose tissue in healthy men. N Engl J Med 360, 1500-1508.

4. Virtanen KA, Lidell ME, Orava J, et al. (2009) Functional brown adipose tissue in healthy adults. $N$ Engl J Med 360 $1518-1525$.

5. Yoneshiro T, Aita S, Matsushita M, et al. (2011) Brown adipose tissue, whole-body energy expenditure, and thermogenesis in healthy adult men. Obesity 19, 13-16.

6. Yoneshiro T, Aita S, Matsushita M, et al. (2011) Age-related decrease in cold-activated brown adipose tissue and accumulation of body fat in healthy humans. Obesity $\mathbf{1 9}$, $1755-1760$.

7. Kawada T, Watanabe T, Takaishi T, et al. (1986) Capsaicininduced beta-adrenergic action on energy metabolism in rats: influence of capsaicin on oxygen consumption, the respiratory quotient, and substrate utilization. Proc Soc Exp Biol Med 183, 250-256.

8. Kawada T, Hagihara K \& Iwai K (1986) Effects of capsaicin on lipid metabolism in rats fed a high fat diet. J Nutr $\mathbf{1 8 3}$ $1272-1278$. 
9. Yoshioka M, Lim K, Kikuzato S, et al. (1995) Effects of red-pepper diet on the energy metabolism in men. $J$ Nutr Sci Vitaminol 41, 647-656.

10. Lejeune MP, Kovacs EM \& Westerterp-Plantenga MS (2003) Effects of capsaicin on substrate oxidation and weight maintenance after modest body-weight loss in human subjects. Br J Nutr 90, 651-659.

11. Ludy MJ, Moore GE \& Mattes RD (2012) The effects of capsaicin and capsiate on energy balance: critical review and metaanalyses of studies in humans. Chem Senses 37, 103-121.

12. Yoneshiro T, Aita S, Kawai Y, et al. (2012) Non-pungent capsaicin analogs (capsinoids) increase energy expenditure through the activation of brown adipose tissue in humans. Am J Clin Nutr 95, 845-850.

13. Ono K, Tsukamoto-Yasui M, Hara-Kimura Y, et al. (2011) Intragastric administration of capsiate, a transient receptor potential channel agonist, triggers thermogenic sympathetic responses. J Appl Physiol 110, 789-798.

14. Kawabata F, Inoue N, Masamoto $\mathrm{Y}$, et al. (2009) Nonpungent capsaicin analogs (capsinoids) increase metabolic rate and enhance thermogenesis via gastrointestinal TRPV1 in mice. Biosci Biotechnol Biochem 73, 2690-2697.

15. Akendengué B \& Louis AM (1994) Medicinal plants used by the Masango people in Gabon. J Ethnopharmacol 41, 193-200.

16. Umukoro S \& Ashorobi RB (2001) Effect of Aframomum melegueta seed extract on thermal pain and on carrageenininduced oedema. Nig QJ Hosp Med 11, 220-225.
17. Connell DW (1970) Natural pungent compounds. III. Paradols and associated compounds. Aust I Chem 23 , 369-376.

18. Connell DW \& McLachlan R (1972) Natural pungent compounds. IV. Examination of the gingerols, shogaols, paradols, and related compounds by thin-layer and gas chromatography. J Chromatogr A 61, 29-35.

19. Tackie AN, Dwuma-Badu D, Ayim JSK, et al. (1975) Constituents of West African medicinal plants. VIII. Hydroxyphenylalkanones from Amomum melegueta. Phytochemistry 14, 853-854

20. Riera CE, Menozzi-Smarrito C, Affolter M, et al. (2009) Compounds from Sichuan and Melegueta peppers activate, covalently and non-covalently, TRPA1 and TRPV1 channels. Br J Pharmacol 157, 1398-1409.

21. Iwami M, Mahmoud FA, Shiina T, et al. (2011) Extract of grains of paradise and its active principle 6-paradol trigger thermogenesis of brown adipose tissue in rats. Auton Neurosci 161, 63-67.

22. Morera E, Petrocellis De L, Morera L, et al. (2012) Synthesis and biological evaluation of [6]-gingerol analogues as transient receptor potential channel TRPV1 and TRPA1 modulators. Bioorg Med Chem Lett 22, 1674-1677.

23. Kawada T, Shun K, Yoshifumi H, et al. (1998) Fish (Bonito) oil supplementation enhance the expression of uncoupling protein in brown adipose tissue of rat. J Agric Food Chem 46, 1225-1227. 